\title{
Automated microfluidic sample-preparation platform for high-throughput structural investigation of proteins by small-angle X-ray scattering
}

Lafleur, Josiane P.; Snakenborg, Detlef; Nielsen, Søren Skou; Møller, Magda; Toft, Katrine N.; Menzel, Andreas; Jacobsen, Jes K.; Vestergaard, Bente; Arleth, Lise; Kutter, Jörg Peter

Published in:

Journal of Applied Crystallography

Link to article, DOI:

$10.1107 /$ S0021889811030068

Publication date:

2011

Document Version

Publisher's PDF, also known as Version of record

Link back to DTU Orbit

Citation $(A P A)$ :

Lafleur, J. P., Snakenborg, D., Nielsen, S. S., Møller, M., Toft, K. N., Menzel, A., Jacobsen, J. K., Vestergaard, B., Arleth, L., \& Kutter, J. P. (2011). Automated microfluidic sample-preparation platform for high-throughput structural investigation of proteins by small-angle X-ray scattering. Journal of Applied Crystallography, 44(5), 1090-1099. https://doi.org/10.1107/S0021889811030068

\section{General rights}

Copyright and moral rights for the publications made accessible in the public portal are retained by the authors and/or other copyright owners and it is a condition of accessing publications that users recognise and abide by the legal requirements associated with these rights.

- Users may download and print one copy of any publication from the public portal for the purpose of private study or research.

- You may not further distribute the material or use it for any profit-making activity or commercial gain

- You may freely distribute the URL identifying the publication in the public portal 
Journal of

\section{Applied Crystallography}

ISSN 0021-8898

Received 21 March 2011

Accepted 25 July 2011

C 2011 International Union of Crystallography Printed in Singapore - all rights reserved

\section{Automated microfluidic sample-preparation platform for high-throughput structural investigation of proteins by small-angle X-ray scattering}

\author{
Josiane P. Lafleur, ${ }^{a}$ Detlef Snakenborg, ${ }^{a}$ Søren S. Nielsen, ${ }^{\text {a,b }}$ Magda Møller, ${ }^{c}$ \\ Katrine N. Toft, ${ }^{c}$ Andreas Menzel, ${ }^{\text {d }}$ Jes K. Jacobsen, ${ }^{\text {e }}$ Bente Vestergaard, ${ }^{c}$ Lise \\ Arleth $^{b}$ and Jörg P. Kutter ${ }^{\mathrm{a} *}$ \\ aDepartment of Micro and Nanotechnology, Technical University of Denmark, Denmark, \\ ${ }^{\mathbf{b}}$ Department of Basic Sciences and Environment, University of Copenhagen, Denmark, \\ ${ }^{\mathbf{c}}$ Department of Medicinal Chemistry, University of Copenhagen, Denmark, ${ }^{\mathbf{d}}$ Synchrotron Radiation

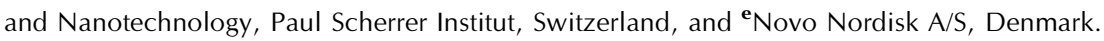 \\ Correspondence e-mail: joerg.kutter@nanotech.dtu.dk
}

\begin{abstract}
A new microfluidic sample-preparation system is presented for the structural investigation of proteins using small-angle X-ray scattering (SAXS) at synchrotrons. The system includes hardware and software features for precise fluidic control, sample mixing by diffusion, automated X-ray exposure control, UV absorbance measurements and automated data analysis. As little as $15 \mu \mathrm{l}$ of sample is required to perform a complete analysis cycle, including sample mixing, SAXS measurement, continuous UV absorbance measurements, and cleaning of the channels and X-ray cell with buffer. The complete analysis cycle can be performed in less than 3 min. Bovine serum albumin was used as a model protein to characterize the mixing efficiency and sample consumption of the system. The N2 fragment of an adaptor protein (p120-RasGAP) was used to demonstrate how the device can be used to survey the structural space of a protein by screening a wide set of conditions using high-throughput techniques.
\end{abstract}

\section{Introduction}

The ability of small-angle X-ray scattering (SAXS) to provide low-resolution structural information on proteins directly in solution makes it the tool of choice for analysing the structural changes of proteins in response to variations in their chemical environment. However, in a traditional SAXS experiment only part of the experimental space of the protein can be investigated because of practical constraints. The experimental conditions that may promote structural changes in a given protein are often unknown prior to the experiment. Thus, it is necessary to investigate the structural state of the protein under a large number of experimental conditions. At the same time, not only is the available quantity of many macromolecules of biological interest limited, but the frequency and duration of experimental campaigns at synchrotron facilities are restricted. Furthermore, at most synchrotron beamlines, sample changing tends to be time consuming and, as a result, only a few experimental conditions can be investigated within the allocated time. As a consequence, when pre-defined samples have to be prepared and transferred to the sample chamber manually prior to analysis, only a fraction of the structural space of a given biomacromolecular system is investigated. Efficient sample changing, in terms of both volume and time, is therefore of paramount importance for exploring the rich structural space of many biomacromolecular systems.

Key requirements to maximize the usefulness of structural biology methods include the ability to operate in situ under variable and close-to-native experimental environments, the possibility of high-throughput studies, and the rapid generation of structural models (Svergun, 2010). The limitations of current SAXS methods have prompted a significant interest in developing robust high-throughput fully automated methods for SAXS analysis which will fulfil these requirements. Recent efforts have focused mainly on sample-changing robots (Round et al., 2008; Hura et al., 2009; David \& Pérez, 2009) in order to automate experiments, minimize sample consumption, save time and minimize human error. In terms of speed and sample consumption, the most efficient method published so far consists of using a pipetting robot to transfer $12 \mu \mathrm{l}$ predefined samples from a 96-well plate to a SAXS sample cell (Hura et al., 2009). However, this approach lacks the significant advantages offered by microfluidic devices. Firstly, with microfluidic devices the sample conditions can easily be changed 'on the fly' in response to changes in the observed signal. Secondly, the inherent modular construction of microfluidic devices offers the possibility of a synergistic 
combination with complementary sample-handling or characterization units.

Pollack et al. $(1999,2001)$ pioneered the combination of a simple microfluidic diffusion-based mixer with SAXS measurements in a flow-through cell to measure the folding kinetics of proteins after a $\mathrm{pH}$ jump. Akiyama et al. (2002) and Uzawa et al. $(2004,2006)$ have also developed a continuousflow mixing device to study the folding dynamics of several proteins by SAXS. Panine et al. (2006) reviewed the status and limitations of these continuous-flow microfluidic mixing devices for SAXS. Marmiroli et al. $(2009,2010)$ used a free-jet micromixer to study the kinetics of nanoparticle formation using time-resolved SAXS. All these rapid-mixing devices were designed specifically to study folding events or reaction kinetics with submillisecond time resolution but did not offer any flexibility in terms of experimental design. Otten et al. (2005) have also used microfluidics to investigate soft matter by SAXS. Microfluidic devices can also be used to perform very specific functions. For example, Martel and co-workers (Martel, Burghammer, Davies, Di Cola, Panine et al., 2008; Martel, Burghammer, Davies, Di Cola, Vendrely \& Riekel, 2008) have studied silk fibre assembly by SAXS in a concentric flow microfluidic cell designed to mimic the geometry of the spinning duct of the silkworm. Nevertheless, the search for relevant structural states in proteins is not a straightforward process and often requires the investigation of many different sample conditions, a tedious procedure which calls for the development of a very versatile high-throughput microfluidic device. Toft et al. (2008) reported a high-throughput device for protein structural analysis on a relatively simple microfluidic front-end named the bioXTAS chip. This chip enabled automated mixing of samples by diffusion, and featured a 200 or $500 \mathrm{nl} \mathrm{X-ray} \mathrm{chamber.} \mathrm{Protein} \mathrm{consumption} \mathrm{was} \mathrm{limited} \mathrm{to}$ $36 \mu \mathrm{l}$, with a total cycle time, including protein and buffer measurements, of $28 \mathrm{~min}$. However, fluidic control was compromised by the presence of fluidic compliance and the absence of valves.

The new version of the bioXTAS chip presented here features several additions, transforming the initial design from a simple microfluidic front-end to a true 'lab-on-a-chip' device including all the necessary steps of the analytical process, from sample preparation to detection and semi-automated data analysis. Protein dead-volume is minimized by the addition of an on-chip sample reservoir, which allows the injection of small sample aliquots using buffer or an immiscible fluid as the carrying fluid. Motor-controlled rotary valves reduce the impact of fluidic capacitance and allow accurate on-chip sample preparation. Sample progress and protein concentration are continuously monitored by UV absorbance through integrated optical fibres. Open-source software programs developed in-house provide integrated synchronized control of the beamline X-ray shutter systems and in situ automated two-dimensional data reduction and preliminary analysis of the isotropic SAXS data (Nielsen et al., 2009). The synergistic combination of the microfluidics capabilities with the automation and data-processing software allows the user to make on-the-fly adjustments to the experimental conditions based on the observed data, providing SAXS beamline users with an unprecedented flexibility and throughput for the analysis of protein systems.

The function of many protein systems is connected with structural flexibility and/or the formation of new inter- or intramolecular protein contacts. For these protein systems, it is of great relevance to develop high-throughput devices capable of providing information on their three-dimensional quaternary structures and thus gain insight into their structure-function relationship. In this paper, a GTPase-activating protein (p120 RasGAP; Miao et al., 1996; Pamonsinlapatham et al., 2009) was used as a model to demonstrate how the bioXTAS chip can facilitate the exploration of the relevant structural space of a protein. This protein, known as an adaptor protein, possesses several conserved domains mediating protein-protein interactions. Adaptor protein systems are structurally interesting because they change their shape and complex state when participating in cell-signalling events (Flynn, 2001). In particular, p120 RasGAP plays a central role in the inactivation of the proto-oncogenic protein p21Ras, associated with various known cancer forms (Michod et al., 2004; Pamonsinlapatham et al., 2008). The $N$-terminal part of p120 RasGAP exists naturally in the cell as the N2 fragment (Yang \& Widmann, 2001). This N2 fragment forms protein complexes during cell signalling and is a relevant therapeutic drug target (Ellis et al., 1990; Yang et al., 2005). The exploration of the unknown structural space of the N2 fragment of p120 RasGAP is therefore potentially therapeutically relevant, because it is suggested that structural changes mediate the relevant protein-protein contacts.

\section{Methods}

\subsection{Microfluidic chip design and fabrication}

2.1.1. Microfluidic device fabrication. The bioXTAS labon-a-chip system consists of two modules, the mixing chip and the sample-detection chip.

The mixing chip, illustrated in Fig. 1(a), supports the mixing channels and rotor valves (marked A and B in Fig. 1). The mixing chip and rotor valves consist of a $3 \mathrm{~mm}$-thick poly(methyl methacrylate) (PMMA) substrate featuring $100 \times$ $200 \mu \mathrm{m}$ micromilled channels. PMMA was used as the substrate material because of its machinability. The fabrication resulted in burr-free channels, and the sample chambers and chips could be easily mass-produced. The channels are sealed by bonding with a $1 \mathrm{~mm}$-thick PMMA lid featuring microdrilled inlets and outlets. Bonding was achieved by exposing the PMMA parts to UV light $\left(30 \mathrm{~mW} \mathrm{~cm}^{-2}\right)$ for $2 \mathrm{~min}$. The exposed parts were then aligned and bonded thermally $(10 \mathrm{kN}$ applied pressure for the mixing chip or $1 \mathrm{kN}$ each for the rotors, at $358 \mathrm{~K}$ for $1 \mathrm{~h}$ ).

The sample-detection chip, illustrated in Fig. 1(b), consists of a $1.7 \mathrm{~mm}$-thick polystyrene (PS) substrate featuring $250 \times$ $400 \mu \mathrm{m}$ micromilled channels. The detection area of the sample chip, outlined in red in Fig. 1, consists of a cylindrical $\mathrm{X}$-ray cell (marked C in Fig. 1; $800 \mu \mathrm{m}$ diameter, $1.7 \mathrm{~mm}$ deep) 
for SAXS analyses and a $2 \mathrm{~mm}$-long UV cell (marked D in Fig. 1). The channels and X-ray chamber are sealed on both sides of the chip by a $125 \mu$ m-thick PS film bonded thermally ( $3 \mathrm{kN}$ applied pressure at $343 \mathrm{~K}$ for $1 \mathrm{~h}$ ). The choice of PS as the window material for the $\mathrm{X}$-ray measurements was supported by tests performed with a rotating-anode X-ray source on Bruker Nanostar equipment (Toft et al., 2008). After bonding, the optical fibres $(200 \mu \mathrm{m}$ core, FVP 200220240; Polymicro Technologies, Phoenix, Arizona, USA) were inserted through two micromilled grooves on either side of the UV chamber (marked $\mathrm{E}$ in Fig. 1). The grooves were then filled with an optical adhesive (NOA61; Norland Products Inc., Cranbury, New Jersey, USA) by capillary action and cured for $10 \mathrm{~s}$ under UV light (UV75; Thorlabs, Newton, New Jersey, USA). The sample-detection chip inlet channel (coloured purple in Fig. 1) is located on the front of the chip, while the waste channel (the dashed purple line in Fig. 1) and waste outlet (marked F in Fig. 1) are located on the back of the chip.

Two alignment notches fitted with dowel pins allow the quick assembly of the sample-detection and mixing chips. Poly(dimethyl siloxane) (PDMS) O-rings, made in-house, ensure a tight seal between the two clamped chips.

2.1.2. Rotor valves and protein reservoir. The three PMMA rotational valves (marked A in Fig. 1) can be turned from the on to the off position using stepper motors and position encoders for correct positioning. Additionally, the rotational

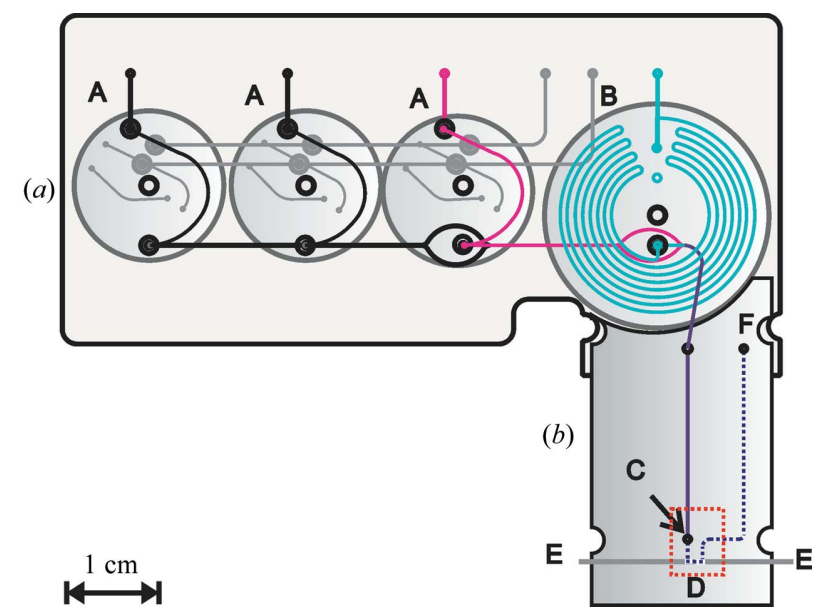

Figure 1

Microfluidic chip design. The complete system consists of $(a)$ a PMMA mixing chip featuring valves and inlets, and $(b)$ a PS sample-detection chip where spectroscopic measurements are performed. Buffers and reagents can be introduced through three separate inlets, each equipped with a rotary valve (A). The rotary valves allow switching between four states, connecting three different inlets with a mixing channel, and an additional closed position. A larger rotary valve (B) doubles as an onchip protein reservoir. For protein dilution, the buffer channel (magenta line) bifurcates around the protein inlet (blue) to laminate the protein sample on two sides (purple). SAXS and UV absorbance measurements can be performed simultaneously on the mixed sample in the detection area of the chip (outlined in red). SAXS measurements are obtained directly through the thin PS covers of the X-ray cell (C), while light is guided to and from the UV absorbance cell (D) by two integrated optical fibres (E). The sample exits the chip through the waste channel (dashed purple line) and outlet (F), both located on the back of the chip. valves can provide a connection from three different inlets to the main channel for experiments requiring more fluidic components. The larger rotational valve (marked B in Fig. 1) features an on-chip protein reservoir in the form of a meandering channel (turquoise). In order to reduce protein dead-volume, small samples can be injected into the storage channel of the valve and small aliquots can then be pushed into the mixing chip using an immiscible fluid. Flow rates are controlled by custom-built miniaturized and automated syringe pumps. The combination of different component flow rates is used to adjust the sample composition to the desired specifications.

2.1.3. Automation software, hardware and beamline control. The custom-developed control software ECON+ was developed in the Python programming language. ECON+ communicates with or directly controls all the hardware. Unlike the automation setup used by Toft and co-workers, which used commercial syringe pumps and lacked on-chip valves (Toft et al., 2008), custom hardware was developed to improve fluidic control. Syringe-pump stages were built inhouse and consisted of four stepper motors controlled by sending pulses to a microstepping motor controller. The four PMMA rotary valves were controlled by individual stepper motors and four potentiometers allowed their accurate positioning.

Fig. 2 provides a schematic illustration of the automation setup. Through a graphical user interface (GUI), the ECON+ software provides the user with the ability to control the pumps and valves individually and to pre-program fully automated series of measurements. A laptop running $\mathrm{ECON+}$ controls the valve motors and the syringe-pump stage motors through simple messages sent through a serial connection to the control box.

$\mathrm{X}$-ray exposures were initiated automatically through ECON+. Network communication to the SPEC beamline control server was achieved using the Python library developed by the European Synchrotron Radiation Facility

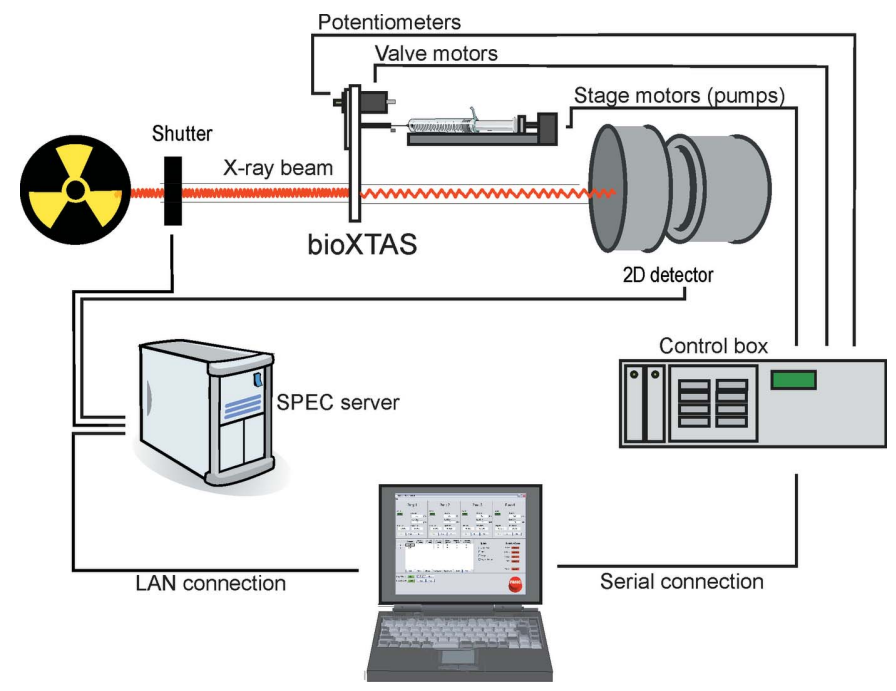

Figure 2

Schematic diagram of the automation setup. 
(ESRF-Bliss Framework Project, 2006). ECON+ allows the user to choose between three operational modes. Measurements can be acquired at stopped-flow, in which the valves are closed and pumps stopped after mixing of the sample, continuous-flow, where the valves remain open and fresh sample is continuously supplied during exposure and data acquisition, or delayed exposure, in which the exposure is initiated a certain time after mixing to allow equilibration. More details on the automation software and schematics for the electronic layout of the controller boards are given by Nielsen (2010).

\subsection{Sample preparation}

2.2.1. Expression and purification of the $\mathrm{N} 2$ fragment. Plasmid pASK-IBA13+ enclosing cDNA encoding (kindly provided by Ole Kristensen, Department of Medicinal Chemistry, University of Copenhagen, Denmark) was used for the production of the p120 RasGAP N2 fragment with attached strep tag (positions 176-444, SWISS-PROT database No. P209336; Bairoch \& Apweiler, 2000). Fresh protein samples were prepared prior to the experiment and kept refrigerated. The protein was expressed in Escherichia coli BL21(DE3) pLysS cells. The harvested cells were suspended in $100 \mathrm{~m} M$ Tris- $\mathrm{HCl} / 150 \mathrm{~m} M \mathrm{NaCl} / 1 \mathrm{~m} M$ EDTA buffer. The cell supernatant was loaded onto a strep-tag column and the purification was conducted according to the standard protocol (strep-tag purification protocol, IBA BioTAGnology, Göttingen, Germany). The strep tag was removed by overnight incubation with thrombin. The $\mathrm{N} 2$ fragment protein was dialysed into two different buffer solutions for a minimum of $48 \mathrm{~h}$ : non-reducing buffer, $10 \mathrm{~m} M$ Tris- $\mathrm{HCl} / 100 \mathrm{~m} M \mathrm{NaCl}$ buffer $\mathrm{pH} 8$, and reducing buffer, $10 \mathrm{~m} M$ Tris- $\mathrm{HCl} / 100 \mathrm{~m} M$ $\mathrm{NaCl}$ buffer/0.1 $\mathrm{m} M$ TCEP $\mathrm{pH} 8$.

2.2.2. Sample preparation. Bovine serum albumin (BSA; Sigma-Aldrich, St Louis, Missouri, USA) stock solutions were prepared in $25 \mathrm{~m} M$ HEPES buffer. BSA stock concentrations were $9 \mathrm{mg} \mathrm{ml}^{-1}$. All protein samples were centrifuged for $20 \mathrm{~min}(9056 \mathrm{~g}$ at $277 \mathrm{~K})$ prior to SAXS data collection and kept refrigerated. During measurements, protein and buffer samples were kept in $5 \mathrm{ml}$ glass syringes, mounted on pumps connected to the bioXTAS chip. All protein concentrations were determined using a Nanodrop 1000 spectrophotometer (Thermo Fisher Scientific Inc, Wilmington, Delaware, USA) prior to SAXS measurements.

\subsection{Spectroscopic measurements}

2.3.1. SAXS measurements. The SAXS measurements presented here were obtained at the Swiss Light Source (SLS) cSAXS beamline at the Paul Scherrer Institut (Villigen, Switzerland). The SLS synchrotron is a third-generation light source. Data were collected using a Pilatus 2M detector (Henrich et al., 2009; Kraft et al., 2009), which is capable of sustaining an $\sim 30 \mathrm{~Hz}$ full-frame readout rate. The operating range of the cSAXS beamline is $6.2-18.6 \mathrm{keV}$, or $0.7-2 \AA$. SAXS data were acquired in the range $0.0085<q<0.61 \AA^{-1}$, where $q=4 \pi \sin (\theta) / \lambda$ is the scattering vector, $2 \theta$ the scattering angle and $\lambda$ the incident wavelength. An $x y$ translation stage was used to position the sample-detection chip in the X-ray path. The exposure time was set to $4 \mathrm{~s}(5 \times 0.8 \mathrm{~s})$ and the spot size on the sample was $200 \times 400 \mu \mathrm{m}$ (vertical $\times$ horizontal). The beam was fully contained within the detection cell and did not overlap with the PMMA cell walls.

2.3.2. SAXS data processing. A fully open-source software program, bioXTAS RAW, developed in-house (Nielsen et al., 2009), was used for the automated two-dimensional data reduction and preliminary analysis of the isotropic SAXS data. bioXTAS RAW handles all steps of the data reduction and has the ability to normalize the sample data automatically and subtract the buffer signals, and to obtain the pair distance distribution function $p(r)$ using the Bayesian indirect Fourier transform algorithm (Hansen, 2000). The radius of gyration $\mathrm{Rg}$ and the forward scattering intensity $I(0)$ were obtained through the Guinier approach (Guinier \& Fournet, 1955). In the $\mathrm{N} 2$ analyses, all scattering data were buffer-subtracted and normalized with respect to protein concentration. $I(0)$ was used to estimate the molecular weight (MW) of N2 using BSA as an MW standard. The program GNOM (Svergun, 1992) was used for calculating the pair distance distribution functions of the $\mathrm{N} 2$ data and estimation of the maximal distance $\left(D_{\max }\right)$ within the scatterer. The exposure time was set to $5 \times 0.8 \mathrm{~s}$ frames to assess possible radiation damage. Frames where potential damage was observed could be discarded prior to the averaging of individual frames. No radiation damage was detected in the analysis of the N2 data.

2.3.3. UV measurements. Integrated optical fibres were used to transmit the light from the UV source (DLS500; Instrument Systems, Munich, Germany) to the diode array spectrometer (USB 4000; Ocean Optics, Dunedin, Florida, USA), shown as E in Fig. 1, in order to obtain continuous UV absorbance measurements.

\section{Results and discussion}

\section{1. bioXTAS lab-on-a-chip system}

Through the development of custom-built hardware and software controlling all aspects of a SAXS analysis, from precise and accurate sample mixing to data acquisition and processing, the bioXTAS chip developed by Toft et al. (2008) has been transformed from a simple microfluidic front end to a fully automated and integrated lab-on-a-chip device. The microfluidic and spectroscopic components of the bioXTAS lab-on-a-chip system are illustrated in Fig. 3. The lab-on-a-chip is composed of two main sections: a mixing chip, where two to four different components can be mixed by diffusion, and a sample-detection chip, where spectroscopic (X-ray and UV) measurements are performed simultaneously on the mixed sample. UV data are acquired continuously and the complementary information they provide can be used to determine sample concentration, to assess channel clean-up and sample position, and to detect the presence of undesirable bubbles in the system. Precise fluidic control was achieved using syringe pumps built in-house, utilized in conjunction with on-chip 
rotary valves. The volumetric flow rates achievable ranged from $200 \mathrm{nl} \mathrm{min}^{-1}$ when the system was equipped with $1 \mathrm{ml}$ glass syringes to $150 \mu \mathrm{min}^{-1}$ when the system was equipped with $5 \mathrm{ml}$ glass syringes. The protein inlet channel is merged with a split buffer channel, allowing lamination of the protein sample from both sides to enhance mixing by diffusion. The minimum distance from the point of mixing to the point of detection is determined by the channel length required for complete mixing by diffusion at the flow rates used. The modular design allows for easy changing of the sampledetection chip in case of radiation damage at high-brilliance synchrotrons. Light is guided to and from the UV cell by two integrated optical fibres, while X-ray measurements are acquired directly through the thin polymeric film covering the $\mathrm{X}$-ray chamber, so they can both be performed simultaneously

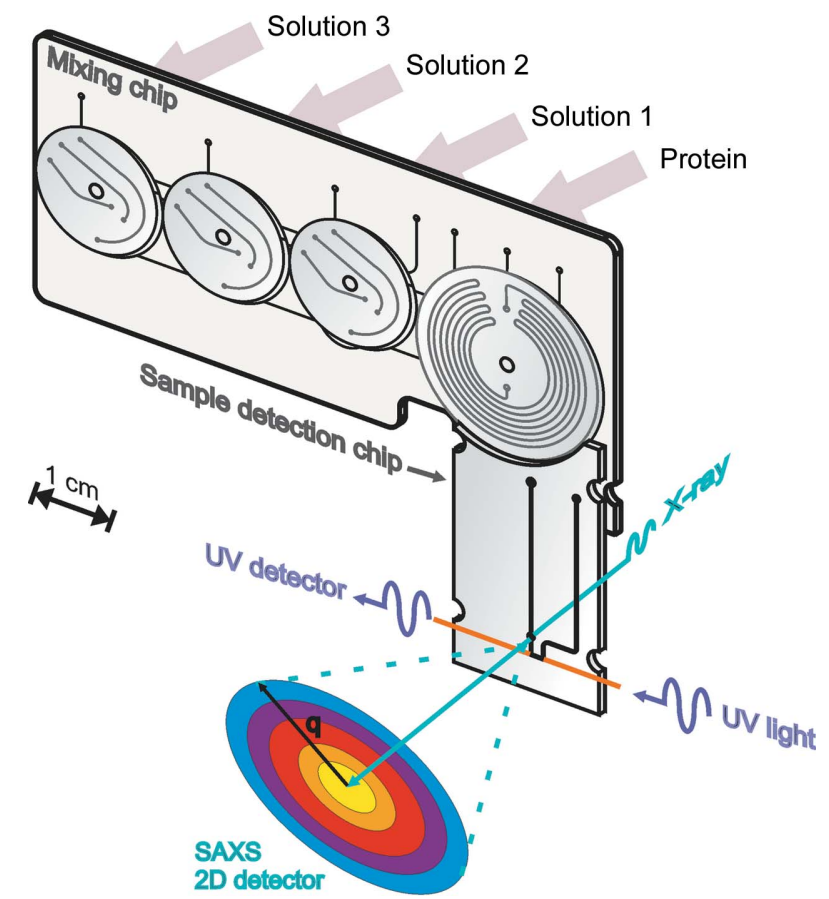

Figure 3

Illustration of the bioXTAS lab-on-a-chip system. The bioXTAS platform allows the continuous variation and monitoring (UV and SAXS) of experimental solution conditions to promote and identify different structural states in proteins in a high-throughput fashion.

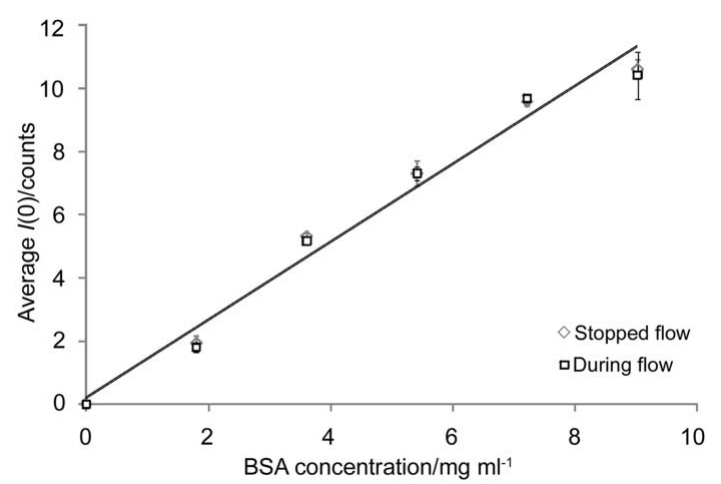

Figure 4

SAXS data recorded for a BSA sample diluted gradually with HEPES buffer (10 $\mu \mathrm{l} \mathrm{min}^{-1}$ combined flow rates, $15 \mu \mathrm{l}$ total injected volume) without interference. A single device, i.e. mixing and sampledetection chips combined, has been used for a period of $24 \mathrm{~h}$ without signs of deterioration or leakage of the seals.

In a typical automated measurement, 15-30 $\mu \mathrm{l}$ samples were mixed and transferred to the detection chambers. The protein valve was then closed to allow cleaning of the chamber with buffer, followed by a background measurement. The composition of the blank can be adjusted as easily and accurately as that of the sample to ensure matrix-matched samples and blanks. During cleaning, the SAXS and UV signals were monitored to ensure proper cleaning. The whole procedure could be completed within 3 min.

3.1.1. Reduction and data-analysis software. As new data come in at high frequencies, especially at high-brilliance thirdgeneration synchrotrons, it is important to be able to analyse the data fast enough for them to be useful in decision making, as well as to identify potential problems, such as protein aggregation, quickly. The bioXTAS RAW software (Nielsen et al., 2009), developed to be used in conjunction with the bioXTAS lab-on-a-chip, was used to read the raw twodimensional SAXS detector output files and plot the data immediately as they were becoming available during the measurement session. The software also allows rapid one-click background subtraction. The instant feedback from the automated reduction and the plotting of new incoming data, as well as the ability to superimpose incoming plots and to average multiple frames, provide an efficient way of quickly analysing the data directly at the beamline.

\subsection{Device characterization}

3.2.1. Reproducibility and accuracy of the automated mixing system. Toft et al. (2008) have already demonstrated that high-quality SAXS data can be obtained using a nanolitre-sized sample chamber. Here, we tested both the reproducibility and the accuracy of the automated mixing system. First, BSA dilution series were performed to assess the quality of the SAXS data collected using the bioXTAS lab-on-a-chip system. The composition of the sample entering the sampledetection chip was varied by adjusting the flow rates of the sample components at the various inlets on the sample-mixing chip using the automated software. Fig. 4 shows that the forward scattering intensity, $I(0)$, is proportional to concentration over the range studied $\left(R^{2}=0.98,1-12 \%\right.$ reproducibility error at the $95 \%$ confidence level, $N=3$ ). Data can be acquired on a continuously renewed sample (during flow) or on an immobilized sample (stopped flow) without any significant difference, as shown in Fig. 4. All subsequent data were acquired on continuously renewed samples. The corresponding scattering data and Guinier range are shown in Supplementary Figs. S1 and S2, respectively.

Second, the accuracy of the automated mixing system was assessed by comparing absorbance readings obtained for BSA protein samples mixed on- and off-chip with HEPES buffer.

\footnotetext{
${ }^{\mathbf{1}}$ Supplementary figures discussed in this paper are available from the $\mathrm{IUCr}$ electronic archives (Reference: EA5144). Services for accessing this material are described at the back of the journal.
} 
Table 1

Comparison of the absorbance measurements for BSA samples mixed on- and off-chip.

\begin{tabular}{lll}
\hline & \multicolumn{2}{l}{ Average absorbance at $280 \mathrm{~nm}$ (arbitrary units) } \\
\cline { 2 - 3 } $\begin{array}{l}\text { BSA concentration } \\
\left(\mathrm{mg} \mathrm{ml}^{-1}\right)\end{array}$ & Mixed on-chip $\dagger$ & Mixed off-chip $\ddagger$ \\
\hline 2.0 & $0.19(1)$ & $0.20(1)$ \\
4.0 & $0.36(1)$ & $0.36(1)$ \\
6.0 & $0.50(2)$ & $0.51(1)$ \\
\hline
\end{tabular}

$\dagger$ Error reported at the $95 \%$ confidence level, $N=6$. $\ddagger$ Error reported at the $95 \%$ confidence level, $N=3$.

For the automated mixing (on-chip mixing), the total flow rate $\left(10 \mu \mathrm{min}^{-1}\right)$ was kept constant while the ratio of buffer flow rate to protein flow rate was varied in order to achieve a range of concentrations. Each concentration was injected for $180 \mathrm{~s}$, resulting in a total sample consumption of $30 \mu \mathrm{l}$. Hand-mixed samples (off-chip mixing) were placed in disposable syringes and manually injected into the sample-detection chip for comparison. The results, presented in Table 1, show that there is no significant difference in the UV intensities recorded when the samples are manually mixed or when they are mixed in a fully automated fashion on the chip. Hence, the bioXTAS microfluidic system provides a precise and accurate means of varying sample composition in a completely automated and high-throughput fashion. Sample composition can be changed on the fly in response to observed experimental results directly from the control room, without the need for the user to access the X-ray hutch where the microfluidic system and samples are located.

3.2.2. Sample consumption. To determine the minimum amount of sample required to replace completely the buffer in the sample cell with a fresh sample, a protein sample was continuously pumped into the microfluidic chip, initially filled with buffer, at various flow rates while the forward scattering was monitored continuously. The sample chamber is considered fully filled with the new sample when the forward scattering becomes constant, resulting in a forward-scattering intensity plateau. Fig. 5(a) shows the reference corrected raw data and Fig. 5(b) the corresponding forward-scattering intensity for BSA injected at $5 \mu \mathrm{min}^{-1}$. As shown by the dotted line in Fig. 5(b), a plateau is reached after the injection of $15 \mu \mathrm{l}$ of sample. After this point, although a slight positive slope can be observed, the error bars for each replicate $(N=3$, 95\% confidence level) start overlapping, and doubling the amount of sample injected does not result in a significant increase in the forward-scattering intensity. Results obtained at flow rates of $5,10,15$ and $20 \mu \mathrm{min}^{-1}$ show that the forward-scattering intensity curves reach a plateau after the injection of $15-25 \mu \mathrm{l}$ of sample, with the highest sample consumption associated with the highest flow rates. Complete cleaning of the BSA-filled chamber required, on average, $22 \mu \mathrm{l}$ of buffer at a flow rate of $10 \mu \mathrm{min}^{-1}$. Therefore, a complete cycle consisting of sample introduction and mixing, X-ray exposure, and cleaning of the channels and sample chamber can be completed in less than $3 \mathrm{~min}$ at a flow rate of $15 \mu \mathrm{min}^{-1}$. These results are comparable to those achieved using current state-of-the-art sampler robots (Hura et al., 2009). The marginal increase in sample consumption is greatly compensated for by the automation of the sample-preparation steps, the complementary UV measurements provided by the modular setup and, last but not least, the added flexibility in modifying the composition of a given sample during the course of an experiment.

\subsection{Screening of the structural space of the $\mathbf{N} 2$ fragment}

The bioXTAS lab-on-a-chip can be used as a highthroughput device to survey the structural space of a protein. We studied the N2 fragment of p120 RasGAP as a model protein to demonstrate the functionality and efficiency of the bioXTAS system. This protein fragment interacts with particular proteins in the cell during cell-signalling events, and structural changes and/or oligomerization are likely to influence protein function. The protein has been investigated under different buffer conditions with and without varying amounts of reducing agents, and, importantly, with varying lengths of equilibration time. The variation in oligomeric state under various conditions is illustrated in Figs. 6 and 7, where the scattering plots $[(a)$ and $(d)]$ and $p(r)$ plots $[(b)$ and $(e)]$

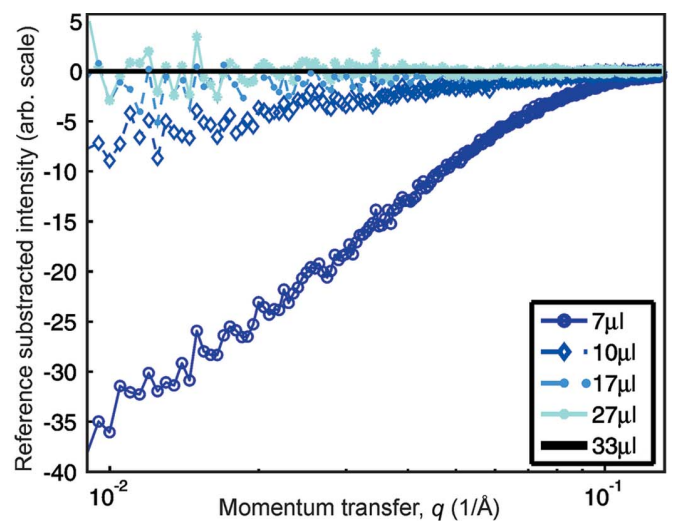

(a)

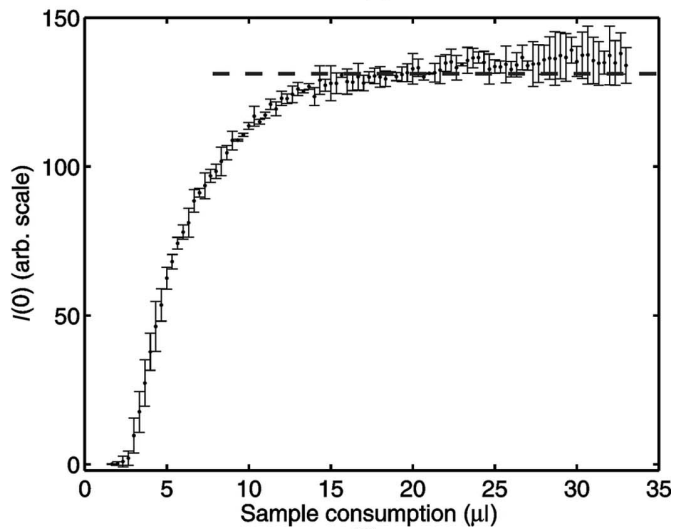

(b)

Figure 5

Sample-consumption determination. BSA solution was continuously injected into the microfluidic cell at a flow rate of $5 \mu \mathrm{min}^{-1}$ for a total injected volume of $33 \mu$ l. (a) Difference between the reference scattering curve obtained after $33 \mu \mathrm{l}$ of injected BSA and the scattering curves obtained during injection. The SAXS intensities are shown as a function of the reciprocal scattering vector $q$. (b) The forward-scatting intensity for the scattering curves, $I(0)$, estimated by the indirect Fourier transform method. 


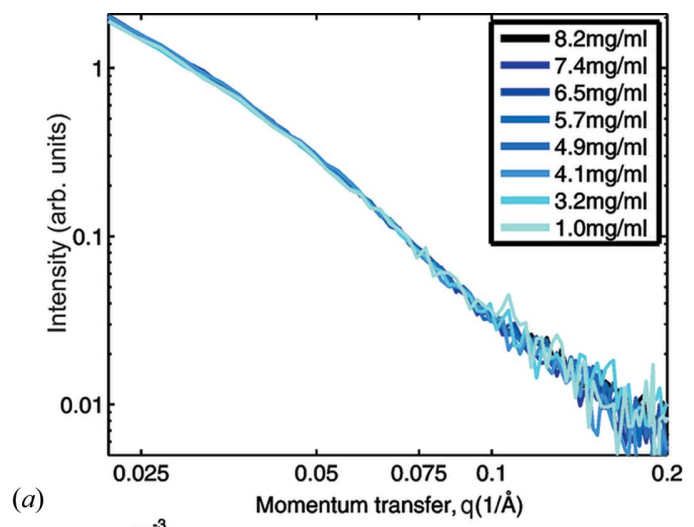

(d)

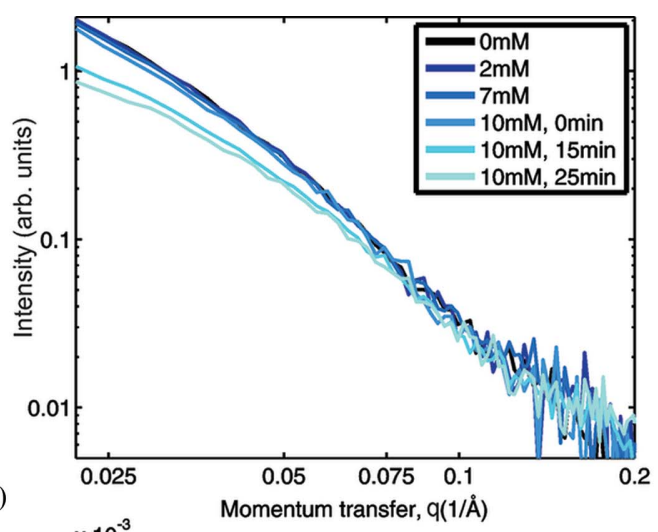

(b)

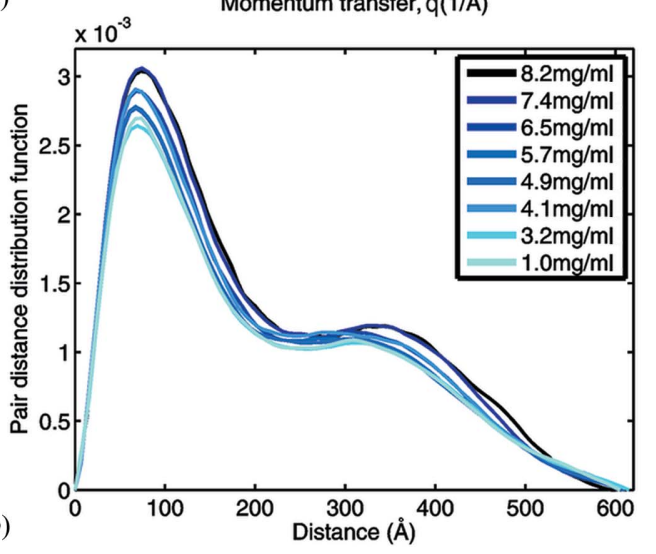

(e)
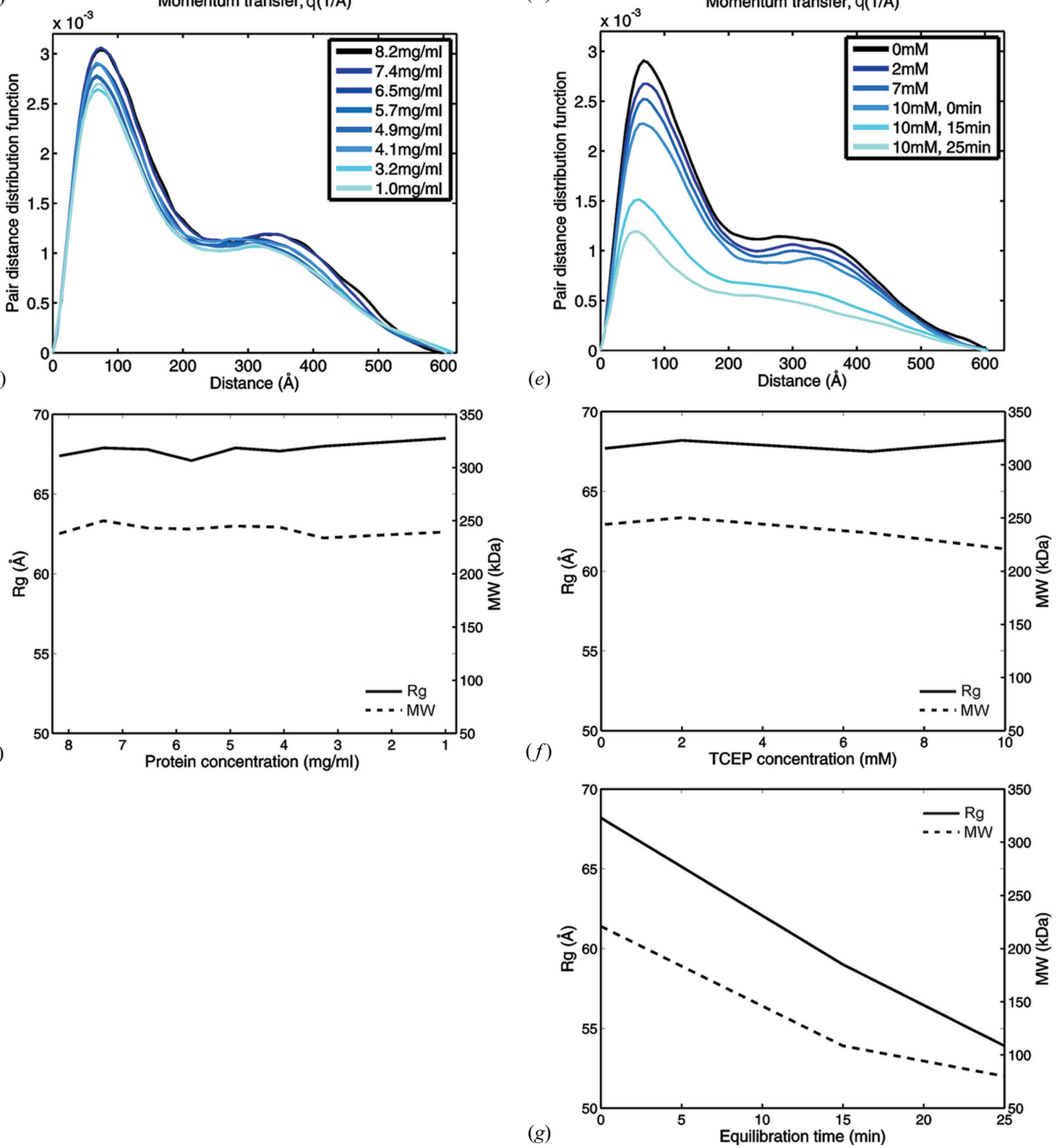

Figure 6

Exploration of the structural space of N2, starting from non-reduced conditions. The left-hand column shows data for N2 dilution with non-reducing buffer: $(a)$ background-subtracted SAXS data normalized to protein concentration, $(b)$ the corresponding pair distance distribution functions, $p(r)$, and (c) estimated radius of gyration $(\mathrm{Rg})$ and molecular weight (MW). The right-hand column shows data for N2 titration with the reducing agent TCEP (0$10 \mathrm{mM}$ ): $(d)$ background-subtracted SAXS data normalized to protein concentration, $(e)$ the corresponding $p(r)$ functions, $(f)$ estimated Rg and MW as a function of TCEP concentration, and $(g)$ estimated Rg and MW for N2 diluted in $10 \mathrm{~m} M$ TCEP after $0-25$ min equilibration. The raw data and $p(r)$ plots for the equilibrated solutions are shown in panels $(d)$ and $(e)$ using lighter blue colours. 
illustrate the relative presence of larger or smaller distances as well as $D_{\max }$. Rg and MW are shown in Figs. 6(c), 6(f) and 6(g) and $7(c)$ and $7(f)$.

Fig. 6 illustrates an exploration of the structural space of N2 fragments dialysed in a non-reducing buffer. As shown in Figs. 6(a)-6(c), the scattering data, $p(r)$ plots and $\mathrm{Rg}$ and $\mathrm{MW}$ values display only minor changes as the protein concentration is varied. This indicates that the oligomeric state of N2 is not significantly affected by variations in protein concentration. The protein solution at constant protein concentration (4.1 $\mathrm{mg} \mathrm{ml}^{-1}$ ) was subsequently titrated with the reducing agent TCEP. The N2 oligomer composition is only slightly affected by the addition of TCEP, as shown in Figs. $6(d)-6(f)$.
However, a clear change in the oligomeric state of $\mathrm{N} 2$ is observed after the sample has been allowed time to equilibrate in $10 \mathrm{~m} M$ TCEP [pale-blue lines in Figs. 6(d), 6(e) and 6(g)]. In Fig. $6(d)$ (pale-blue lines), the scattering curve changes shape, and the falling intensity at lower $q$ values is a clear signature of smaller molecules in the solution. The decrease in area under the $p(r)$ curve shown in Fig. 6(e) (pale-blue lines) indicates correspondingly that the average volume of the particles decreases, although $D_{\max }$ is unchanged. These experiments show that equilibration in the presence of a reducing agent influences the distribution of the oligomeric states of $\mathrm{N} 2$, but also that the size of the highest-order oligomer present in any of the analysed samples remains unchanged.
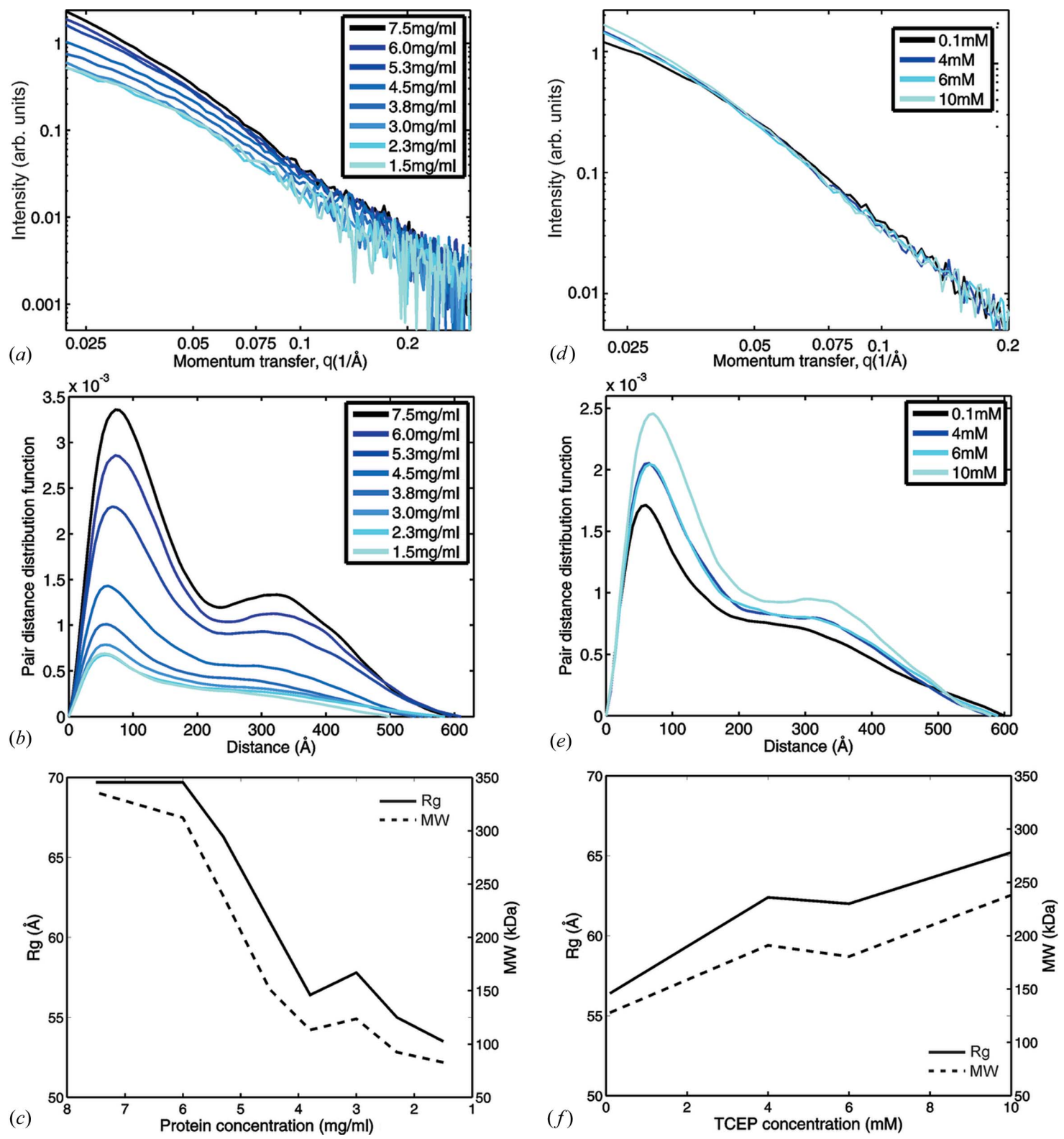

Figure 7

Exploration of N2 protein pre-equilibrated in reducing buffer. The left-hand column shows data for N2 dilution with reducing buffer, $0.1 \mathrm{~m} M$ TCEP: $(a)$ background-subtracted SAXS data normalized to protein concentration, $(b)$ the corresponding $p(r)$ functions, and $(c)$ estimated Rg and MW. The righthand column shows data for $\mathrm{N} 2\left(4.1 \mathrm{mg} \mathrm{ml}^{-1}\right)$ titrated with the reducing agent TCEP $(0-10 \mathrm{mM})$ : $(d)$ background-subtracted SAXS data normalized to protein concentration, $(e)$ the corresponding $p(r)$ functions, and $(f)$ estimated Rg and MW as a function of TCEP concentration. 
Fig. 7 illustrates an exploration of the structural space of N2 fragments dialysed in reducing buffer. A clear decrease in the oligomeric state of $\mathrm{N} 2$ is observed as the protein concentration is decreased by dilution with reducing buffer (Figs. 7a-7c), illustrated by a falling intensity at lower $q$ values. Changes in the overall shape of the scattering curve are also observed. In the $p(r)$ plots (Fig. $7 b$ ), a decrease in $D_{\max }$ indicates that the larger oligomers disappear as the protein concentration is lowered. This trend is also observed in the estimated $\mathrm{Rg}$ and MW shown in Fig. 7(c). We conclude from these results that oligomer formation is reversible, i.e. that an equilibrium between larger and smaller oligomeric proteins exists in solution. Furthermore, Figs. $7(d)-7(f)$ show that, in contrast with samples that were not equilibrated with reducing agent prior to titration, increasing the TCEP concentration leads to a gradual increase in the size of the protein complex.

These results demonstrate the very complex behaviour of the N2 fragment in oligomer formation. If the protein is stored without a reducing agent, it adapts to a structural state where oligomer formation is independent of protein concentration. The oligomeric state is thus not in equilibrium, or at least the equilibrium is strongly shifted towards the oligomeric state. Even when large amounts of reducing agent are added, the oligomeric state changes only slowly towards a smaller protomer. Apparently, including small amounts of reducing agent during storage of the protein causes the protein to adopt a structural state that is in equilibrium between smaller and larger oligomers. At lower concentrations, the oligomer is significantly smaller than in the absence of any reducing agent, while the formation of oligomers at higher protein concentrations supersedes the non-reduced oligomeric state. In such conditions, the oligomeric state is sensitive to the concentration of the reducing agent. It can be concluded that the basic structural state of individual protomers in the oligomers must be different after long-term equilibration with and without reducing agents.

The p120 RasGAP homo-interacting adaptor protein was used in this work to demonstrate the capabilities of the bioXTAS microfluidic setup. The reversible nature of the oligomerization suggests that it could be part of a regulation of p120 RasGAP-mediated cell signalling. Aside from the actual biological function of the protein, the analysis clearly demonstrates that protein structure and/or protein oligomeric state are sensitive to the experimental conditions. This highlights the importance of performing buffer and concentration screens on proteins where oligomerization and structural changes may be of biological relevance. The bioXTAS setup proved to be an ideal platform for this task. These experiments illustrate how rapidly and easily structural changes and oligomerization can be detected by synergistically combining microfluidic sample handling with automated data analysis.

An important characteristic of the bioXTAS samplepreparation platform is its ability to mix several components at once thanks to the many inlets available. This feature offers the possibility of mixing the desired buffers in situ, as shown in the experiments above, extending the capability of the system well beyond simple titration. These experiments also highlight the importance of equilibration time. The versatility of the bioXTAS microfluidic system not only facilitates exact monitoring of the equilibration time but also allows immediate $\mathrm{X}$-ray exposure upon establishment of the desired experimental conditions.

\section{Conclusions}

The flexibility inherent in microfluidic devices, as well as the high level of automation achievable and the greatly reduced sample consumption, can provide an invaluable tool for users at SAXS beamlines. This new lab-on-a-chip system provides the ability to perform many experiments on samples as small as $15 \mu \mathrm{l}$ in a fully automated fashion. This is especially valuable in the context of third-generation synchrotrons, where exposure times are fast and high-throughput microfluidic devices can help maximize the use of the limited beamtime available. The performance of the system is comparable, and in some cases superior, to current methods. The modular design simplifies the integration of various complementary analysis units, such as the UV absorbance measurements demonstrated here. The system was used successfully to screen for changes in the oligomeric state of the $\mathrm{N} 2$ protein as a function of concentration, equilibration time and presence of a reducing agent, thereby elucidating the relevant structural states of the protein system. These results demonstrate the efficiency of the system and its ability to identify experimental conditions that will lead to structural changes in biologically relevant macromolecules.

The authors are grateful for beamtime on the cSAXS beamline at the Swiss Light Source (Villigen, Switzerland), and also for beamtime for pilot experiments on beamline I711 at the MAX-lab Synchrotron (Lund, Sweden) in collaboration with Dr Tomás Plivelic and Dr Yngve Cerenius, and on the SWING beamline at the Synchrotron SOLEIL (Saint-Aubin, France) in collaboration with Dr Gabriel David and Dr Javier Perez. This project was funded by the Danish Research Council for Strategic Research through the NABIIT programme (grant No. 09-060780). Synchrotron access was partly funded through the Danscatt user programme under the Danish Council for Independent Research in Natural Sciences (FNU). The authors thank Per Thor Jonassen (Department of Micro- and Nanotechnologies, Denmark Technical University) for technical support. JPL gratefully acknowledges scholarship support from the Fond Québecois pour la Recherche sur la Nature et les Technologies (FQRNT) and the H. C. Ørsted Postdoctoral Programme.

\section{References}

Akiyama, S., Takahashi, S., Kimura, T., Ishimori, K., Morishima, I., Nishikawa, Y. \& Fujisawa, T. (2002). Proc. Natl Acad. Sci. USA, 99, 1329-1334.

Bairoch, A. \& Apweiler, R. (2000). Nucleic Acids Res. 28, 45-48.

David, G. \& Pérez, J. (2009). J. Appl. Cryst. 42, 892-900.

Ellis, C., Moran, M., McCormick, F. \& Pawson, T. (1990). Nature (London), 343, 377-381. 
ESRF-Bliss Framework Project (2006). Python SpecClient Module, http://www.esrf.eu/UsersAndScience/Experiments/TBS/BLISS/ BLISS-Applications/.

Flynn, D. C. (2001). Oncogene, 20, 6270-6272.

Guinier, A. \& Fournet, G. (1955). Small-Angle Scattering of X-rays, 1st ed. New York: John Wiley.

Hansen, S. (2000). J. Appl. Cryst. 33, 1415-1421.

Henrich, B., Bergamaschi, A., Broennimann, C., Dinapoli, R., Eikenberry, E. F., Johnson, I., Kobas, M., Kraft, P., Mozzanica, A. \& Schmitt, B. (2009). Nucl. Instrum. Methods Phys. Res. Sect. A, 607, 247-249.

Hura, G. L., Menon, A. L., Hammel, M., Rambo, R. P., Poole, F. L. II, Tsutakawa, S. E., Jenney, F. E. Jr, Classen, S., Frankel, K. A., Hopkins, R. C., Yang, S., Scott, J. W., Billard, B. D., Adams, M. W. W. \& Tainer, J. A. (2009). Nat. Methods, 6, 606-612.

Kraft, P., Bergamaschi, A., Broennimann, Ch., Dinapoli, R., Eikenberry, E. F., Henrich, B., Johnson, I., Mozzanica, A., Schlepütz, C. M., Willmott, P. R. \& Schmitt, B. (2009). J. Synchrotron Rad. 16, 368-375.

Marmiroli, B., Grenci, G., Cacho-Nerin, F., Sartori, B., Ferrari, E., Laggner, P., Businaro, L. \& Amenitsch, H. (2009). Lab Chip, 9, 2063-2069.

Marmiroli, B., Grenci, G., Cacho-Nerin, F., Sartori, B., Laggner, P., Businaro, L. \& Amenitsch, H. (2010). Nucl. Instrum. Methods Phys. Res. Sect. B, 268, 329-333.

Martel, A., Burghammer, M., Davies, R., DiCola, E., Panine, P., Salmon, J.-B. \& Riekel, C. (2008). Biomicrofluidics, 2, 024104.

Martel, A., Burghammer, M., Davies, R. J., DiCola, E., Vendrely, C. \& Riekel, C. (2008). J. Am. Chem. Soc. 130, 17070-17074.

Miao, W., Eichelberger, L., Baker, L. \& Marshall, M. S. (1996). J. Biol. Chem. 271, 15322-15329.

Michod, D., Yang, J. Y., Chen, J., Bonny, C. \& Widmann, C. (2004). Oncogene, 23, 8971-8978.

Nielsen, S. S. (2010). PhD Thesis, Technical University of Denmark, Kongens Lyngby, Denmark.
Nielsen, S. S., Toft, K. N., Snakenborg, D., Jeppesen, M. G., Jacobsen, J. K., Vestergaard, B., Kutter, J. P. \& Arleth, L. (2009). J. Appl. Cryst. 42, 959-964.

Otten, A., Köster, S., Struth, B., Snigirev, A. \& Pfohl, T. (2005). J. Synchrotron Rad. 12, 745-750.

Pamonsinlapatham, P. et al. (2008). PLoS ONE, 3, e2902.

Pamonsinlapatham, P., Hadj-Slimane, R., Lepelletier, Y., Allain, B., Toccafondi, M., Garbay, C. \& Raynaud, F. (2009). Biochimie, 91, 320-328.

Panine, P., Finet, S., Weiss, T. M. \& Narayanan, T. (2006). Adv. Colloid Interface Sci. 127, 9-18.

Pollack, L., Tate, M. W., Darnton, N. C., Knight, J. B., Gruner, S. M., Eaton, W. A. \& Austin, R. H. (1999). Proc. Natl Acad. Sci. USA, 96, 10115-10117.

Pollack, L., Tate, M. W., Finnefrock, A. C., Kalidas, C., Trotter, S., Darnton, N. C., Lurio, L., Austin, R. H., Batt, C. A., Gruner, S. M. \& Mochrie, S. G. (2001). Phys. Rev. Lett. 86, 4962-4965.

Round, A. R., Franke, D., Moritz, S., Huchler, R., Fritsche, M., Malthan, D., Klaering, R., Svergun, D. I. \& Roessle, M. (2008). J. Appl. Cryst. 41, 913-917.

Svergun, D. I. (1992). J. Appl. Cryst. 25, 495-503.

Svergun, D. I. (2010). Biol. Chem. 391, 737-743.

Toft, K. N., Vestergaard, B., Nielsen, S. S., Snakenborg, D., Jeppesen, M. G., Jacobsen, J. K., Arleth, L. \& Kutter, J. P. (2008). Anal. Chem. 80, 3648-3654.

Uzawa, T., Akiyama, S., Kimura, T., Takahashi, S., Ishimori, K., Morishima, I. \& Fujisawa, T. (2004). Proc. Natl Acad. Sci. USA, 101, 1171-1176.

Uzawa, T., Kimura, T., Ishimori, K., Morishima, I., Matsui, T., IkedaSaito, M., Takahashi, S., Akiyama, S. \& Fujisawa, T. (2006). J. Mol. Biol. 357, 997-1008.

Yang, J. Y., Walicki, J., Michod, D., Dubuis, G. \& Widmann, C. (2005). Mol. Biol. Cell, 16, 3511-3520.

Yang, J. Y. \& Widmann, C. (2001). Mol. Cell. Biol. 21, 53465358. 